\title{
Effects of the First Decade of Osteoporosis - Therapy with Denosumab (DMAB)
}

ISSN: 2576-8875

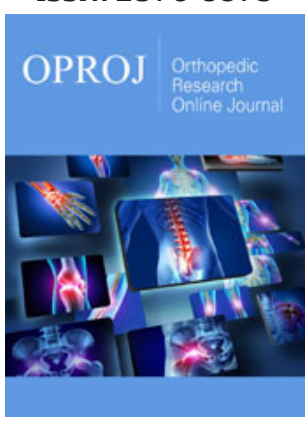

*Corresponding author: Tassilo König, Practice for Orthopaedics / Osteological Specialist Centre DVO, Germany

Submission: 留 February 20, 2020

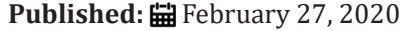

Volume 6 - Issue 5

How to cite this article: Tassilo König* .Effects of the First Decade of Osteoporosis - Therapy with Denosumab (DMAB). Ortho Res Online J. 6(5). OPROJ.000647.2020. DOI: $10.31031 /$ OPROJ.2020.06.000647

Copyright@: Tassilo König, This article is distributed under the terms of the Creative Commons Attribution 4.0 International License, which permits unrestricted use and redistribution provided that the original author and source are credited.

\section{Tassilo König*}

Practice for Orthopaedics / Osteological Specialist Centre DVO, Germany

\section{Summary}

After 10 years of therapy of osteoporosis with the monoclonal antibody Denosumab $\left(\right.$ Prolia $^{\circ}$ ) we look back on the results. Can new fractures be prevented under Denosumab? How long do patients stay on therapy?

The primary goal of osteoporosis therapy is to avoid fractures. Especially major fractures such as radius, femoral neck and vertebral body fractures are accompanied by functional losses, sometimes severe pain and reduced quality of life. Those affected with vertebral body fractures report the most severe pain, often requiring opioid therapy. Fractured osteoporosis patients have an increased risk of death.

In 2010 Denosumab (DMAB) received approval in the EU. Initially approved for the treatment of osteoporosis in postmenopausal women with an increased risk of fractures and for the treatment of bone loss associated with hormone ablation in men with prostate cancer and an increased risk of fractures, the approval was later extended to osteoporosis induced by systemic cortisone therapy and osteoporosis in ankylosing spondylitis due to its proven efficacy.

Denosumab (DMAB) is a fully human monoclonal antibody that inhibits bone resorption, thereby increasing bone mass and reducing the risk of fracture. The antibody binds to the soluble Receptor Activators of Nuclear Factor kappa-B-ligand (RANK- ligand) and thus prevents the activation of its receptor RANK, which controls the maturation of pre-osteoclasts and the function and viability of osteoclasts. This results in a rapid, long-lasting and reversible reduction of bone resorption. In contrast to the likewise anti-resorptive bisphosphonates, DMAB is not stored in the bone.

\section{Patients}

Between 2010-2019 95 women and 27 men were treated with DMAB in our practice and included in the retrospective on the course of DMAB therapy. The average age of the women was 78 , that of the men 75 years. With an average duration of therapy of 3.2 years, this results in a total of about 400 patient years.

The average age of our cohort (DMAB) corresponds to the data from the osteoporosis registry of Germany in that the average age of the women was 77.6 [1]. In Hadji [2] the patients had an average age of 70.6 years.

\section{Specific osteological pre-therapy and prevalent fractures}

$57.7 \%$ of our 122 DMAB patients received a specific osteological pre-therapy in addition to the basic supply of vitamin D and, if required, calcium. Of these, $66 \%$ were first line bisphosphonate (BP) patients (Figure $1 \& 2$ ). 


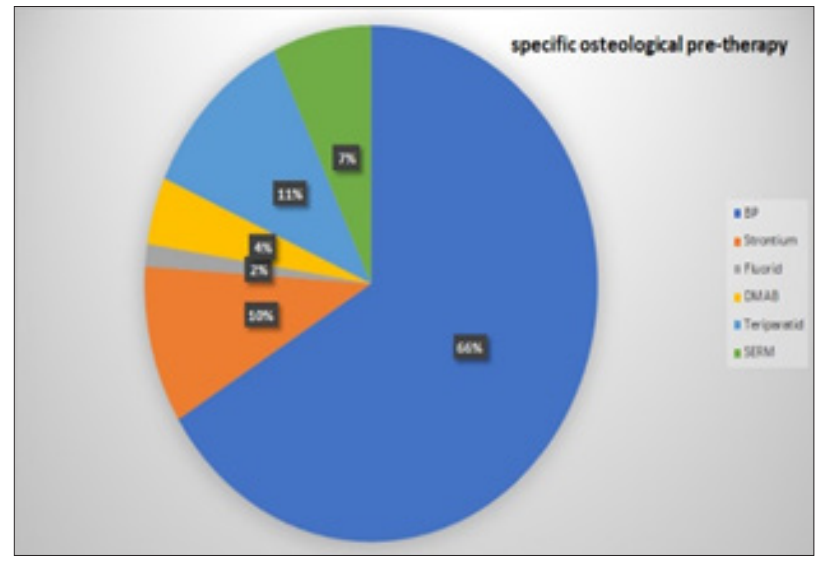

Figure 1:

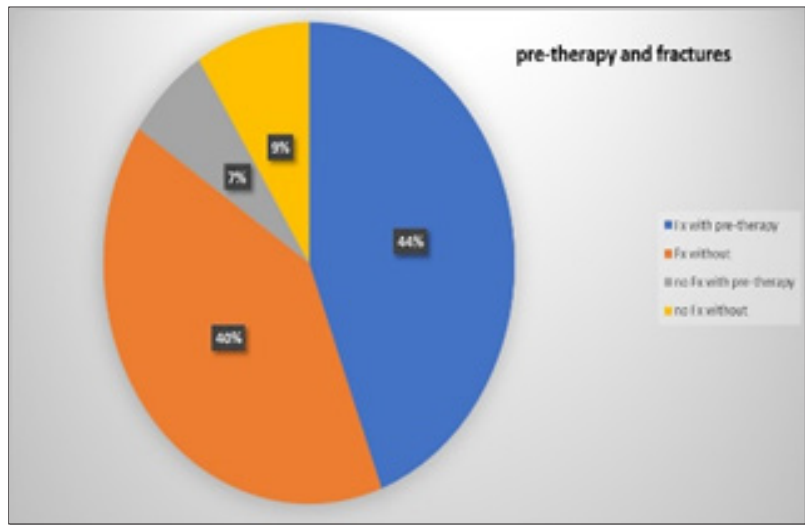

Figure 2:

$84 \%$ of our patients who were adjusted to DMAB therapy were prevalently fractured but only $44 \%$ of these fractured cases received specific pre-therapy! This is still too low a proportion! Also, in the BEST study [3] only $45 \%$ of patients with osteoporosis and fracture received guideline-based therapy. There has not been much improvement so far. Of the 95 women who were recruited by us for DMAB, 64 had prevalent vertebral body fractures and 48 peripheral low-traumatic fractures (Figure 3). In Hadji [2] the rate of prevalent fractures was $50 \%$.

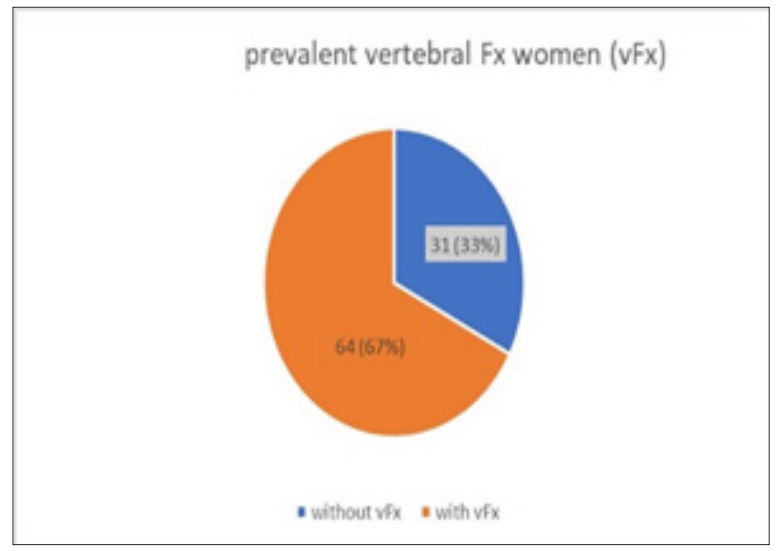

Figure 3:
The 27 men in our group had 21 prevalent vertebral body fractures and 6 peripheral fractures prior to DMAB therapy (Figure 4). As expected, the frequency of multiple fractures increases with age. In women with DMAB indication, most prevalent fractures were grade 3 vertebral body fractures and multiple peripheral fractures $(54 \%)$, while men had grade 3 or multiple fractures in $70 \%$ of cases.

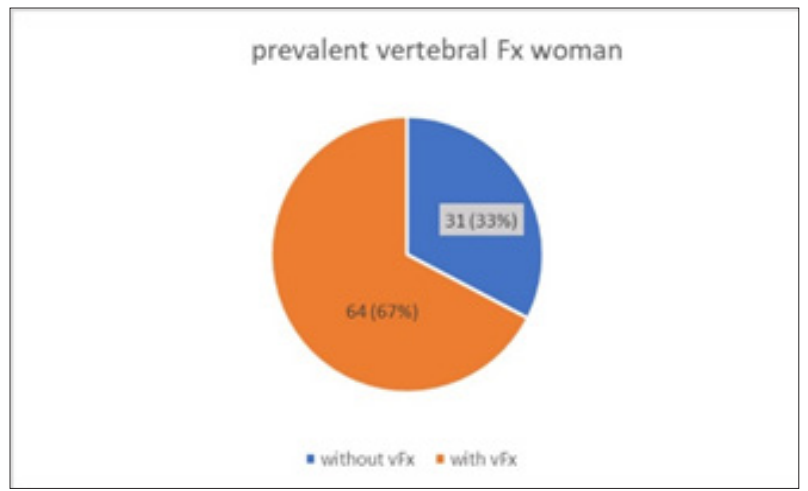

Figure 4:

\section{Bone density before DMAB therapy (DXA measurement)}

The DXA measurement of bone mineral content is still considered the gold standard. DXA -measurements have been carried out in our centre of excellence since 1992. The LSC for the spinal column measurement is $0.028 \mathrm{~g} / \mathrm{cm}^{2}$, for femur total $0.033 \mathrm{~g} /$ $\mathrm{cm}^{2}$.

According to the WHO definition, bone density measurements (DXA) indicate osteoporosis from a T-score of $\leq-2.5$ SD (standard deviation from the mean value of a 20-29 year old woman/men). In order to assess the therapy-relevant 10-year fracture risk, other clinical and anamnestic risk factors (pre-existing fractures, concomitant diseases, long-term drug therapy, femoral neck fracture in family, oral cortisone-therapy etc.) are considered in addition to bone densitometry. Depending on the individual 10year fracture risk determined, specific osteological therapy is initiated even in less severe cases (T-score $\geq-2.5 \mathrm{SD}$ ).

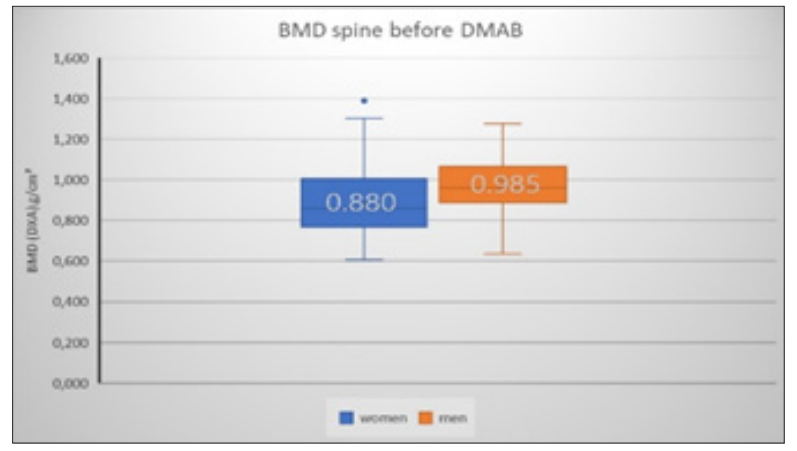

Figure 5:

As expected, in the patients selected for DMAB therapy, men showed the higher initial BMD values for both the spine $(+12 \%)$ and the hips (+4\%) (Figure $5 \& 6$ ). The T-score median for women 
before DMAB therapy was $-2.5 \mathrm{SD}$ at the WS and $-2.3 \mathrm{SD}$ at the hip. For the men the mean T-score was $-1.9 \mathrm{SD}$ at the WS and -2.2 SD at the hip before DMAB therapy.

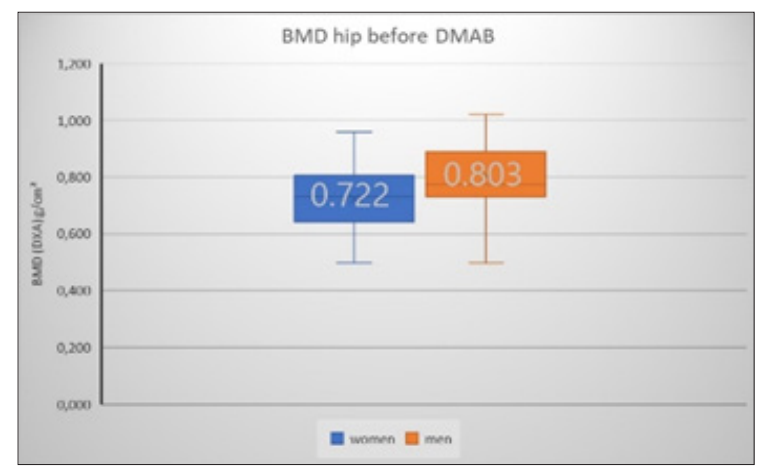

Figure 6:

\section{Change of BMD under DMAB}

It has been shown that bone mineral content (BMD) increases differ depending on whether or not specific anti-resorptive therapy was given prior to DMAB therapy. In the case of no therapy before there was a stronger increase in bone mineral content than in patients who had received anti-resorptive pre-treatment.

For patients under DMAB, the mean annual increase in BMD in the spinal column was $4.5 \%$ p.a. than in those undergoing antiresorptive pre-treatment at $3.1 \%$ p.a. (delta $+1.4 \%$ ). At the total hip, the annual increases were $2.4 \%$ for the therapies and $1.6 \%$ for the specifically pretreated women.

DMAB- therapy was associated with a continuous increase in BMD over the entire therapy period (Figure 7). The cumulative increase in our cohort was $27.2 \%$ for a 7 -year period, higher than in the FREEDOM extension study [4]. The average 8-year gain in the extension study was $18.4 \%$ at the lumbar spine and $8.3 \%$ at the hip.

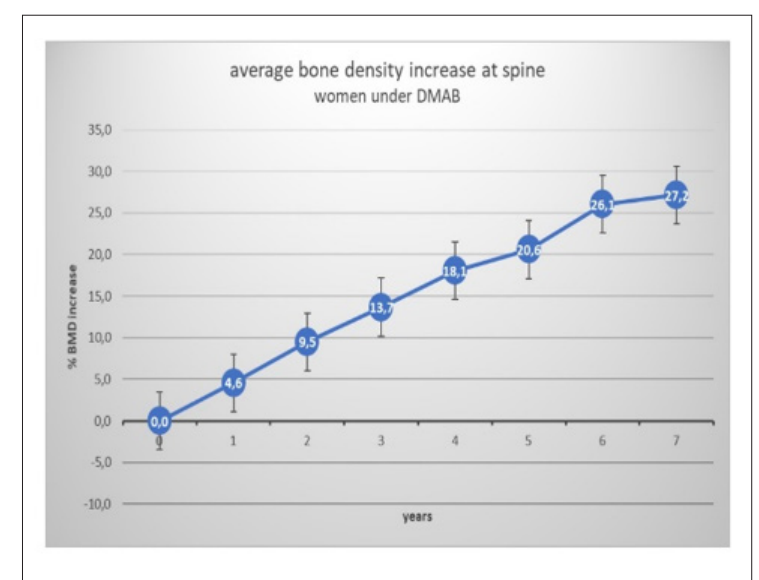

\section{Figure 7:}

In a 6-year analysis [5], a cumulative gain of $13.3 \%$ was measured for the spinal column. In the 10-year extension study, BMD growth was measured at $21.6 \%$ in the lumbar spine and
9.1\% in the hip [6]. It could be shown that the patients had been under DMAB therapy for a median of about 3.2 years and thus had received at least 6 injections of DMAB (Figure 8). After 3 years of therapy 67 of our patients (55\%) were still under DMAB therapy, in the $4^{\text {th }}$ year only $33.6 \%$. At Tuncel [7] 71\% of osteoporosis patients contacted by telephone had not taken any specific osteoporosis therapy after 3 years.

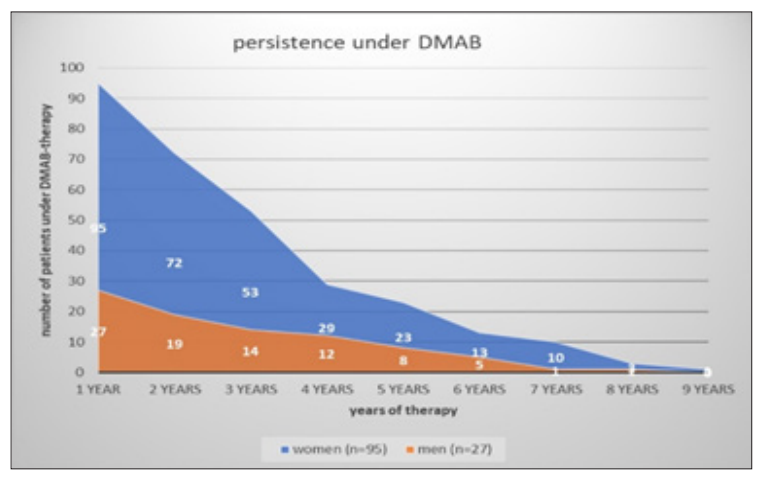

Figure 8:

\section{New fractures under DMAB?}

In our DMAB-treated patients, new vertebral fractures occurred in $8.8 \%$ of cases (Figure 9) and new peripheral fractures in $6 \%$ (Figure 10). With 73\%, new vertebral body fractures occurred predominantly in pre-existing osteoporotic vertebral body fractures.

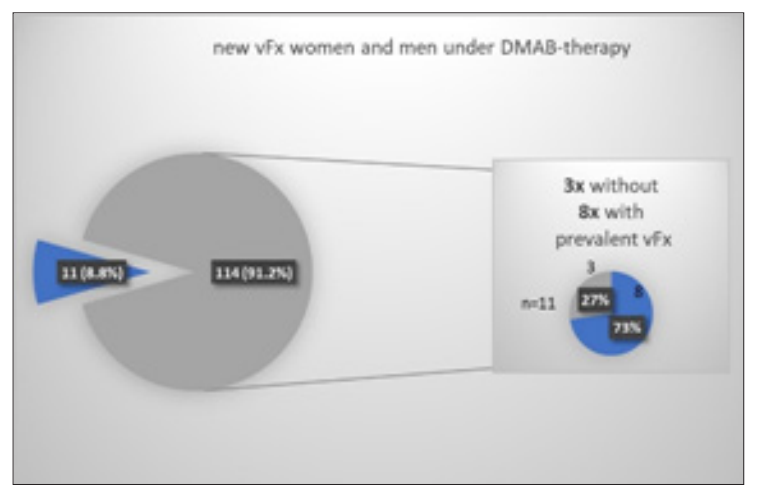

Figure 9:

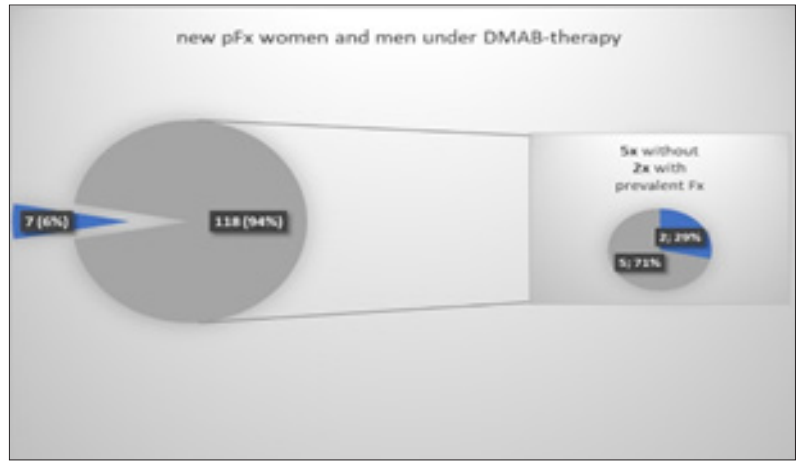

Figure 10: 
The FREEDOM Extension Study indicates a rate of 5.5\% for new spinal fractures up to the $5^{\text {th }}$ year of extension and $6.6 \%$ for peripheral fractures [4].

\section{Therapy pause and therapy interruption}

With high affinity and specificity, DMAB inhibits RANKLmediated osteoclast activity and thus bone resorption. In contrast to the mode of action of bisphosphonates, which are incorporated into the bone and thus have a long retention time, the effect of DMAB is rapidly reversible.

This pharmacological property must be taken into account in case of a therapy break or discontinuation!

The literature reports on the occurrence of multiple fractures in patients after discontinuation of DMAB $[4,8,9]$. A rebound phenomenon is assumed for this effect. After discontinuation of DMAB- therapy, bone density would in some cases fall below the initial value before the start of therapy, which would be associated with an increased risk of fracture!

As an example, I would like to report on three of our patients $(A, B, C)$ in whom the DMAB- therapy was interrupted.

Patient A started DMAB treatment in 03/2012 at the age of 61 years. With a T-score at the lumbar spine of -3.9SD (BMD 0.708g/ $\mathrm{cm}^{2}$ ) and a femoral neck fracture of the mother, there was a strongly increased risk of a 10-year fracture. Vitamin D was $<5.0 \mu \mathrm{g} / \mathrm{l}$, the other values of the basic osteological laboratory were normal. Until $06 / 2015$ there was an increase in BMD of $+9.8 \%$ ( $+3.26 \%$ p.a.). Between the $7^{\text {th }}$ and $8^{\text {th }}$ DMAB- injection, the injection interval was exceeded for 4 months. The DXA control 06/2016 Figure 11 shows a loss of $-6.2 \%$ (BMD $-0.048 \mathrm{~g} / \mathrm{cm}^{2}$; LSC $=0.028 \mathrm{~g} / \mathrm{cm}^{2}$ ). Fortunately, fractures did not occur.

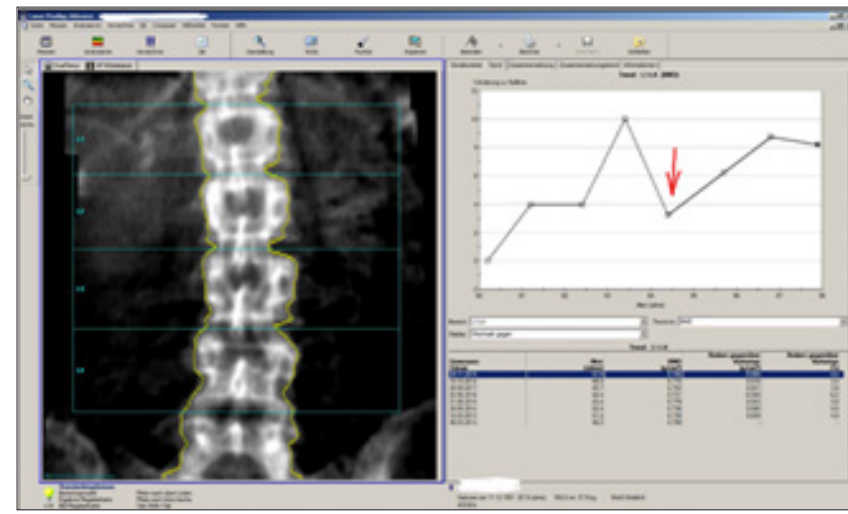

Figure 11:

Patient B started osteoporosis therapy with DMAB at the age of 77 in 10/2010. The T-score of the lumbar spine at this time was $-3.1 S D$. The patient was kidney transplanted. She was accompanied by renal failure grade $2-3$, hyperuricemia, silent inflammation, thyroid carcinoma and recurrent hypocalcemia. Vitamin D was

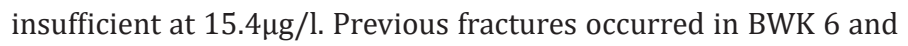
BWK 7, and nine injections of Prolia were given until 07/2014. BMD growth was $+5.8 \%$ at this time. Due to renal insufficiency, there were recurrent prolonged hypocalcemia, a persistent vitamin D deficiency with secondary hyperparathyroidism (iPTH 11pmol/l). The 10th injection of prolia was then given only $02 / 17$, after 31 months. Between 02/2015 and 02/2017, the total loss of bone density was $-11.3 \%$ and thus fell far below the original starting level! After resuming the regular prolia injections, there has been a continuous increase in BMD (Figure 12) of $10.1 \%$ (5\% p.a.). An interim new fracture has not occurred.

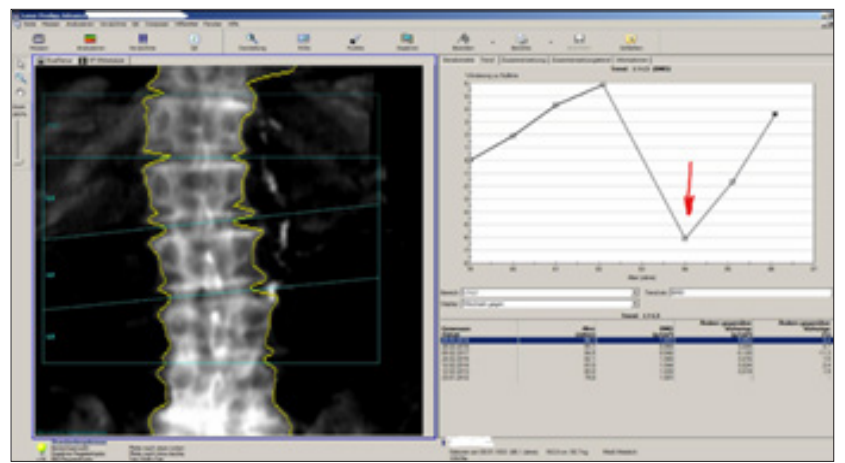

Figure 12:

Patient C began osteological therapy at the age of 55 in 2000 with colleagues who had previously treated her. Initially adjusted to weekly oral bisphosphonates, the patient received prolia injections for a compliance disorder from 2013-2016. After a control DXA measurement (T-score femoral neck right -2.86SD), DMAB was discontinued without specific follow-up therapy because of "good improvement in values and low osteoporosis". After discontinuation, three new WK fractures (LWK2,3,4) occurred in the course of the year, of which LWK2 and LWK3 were kyphoplasted (Figure 13). The patient then suffered a fracture of the radius on the right and fractures of the carpal bones on the right, several fractures of the fingers and toes.

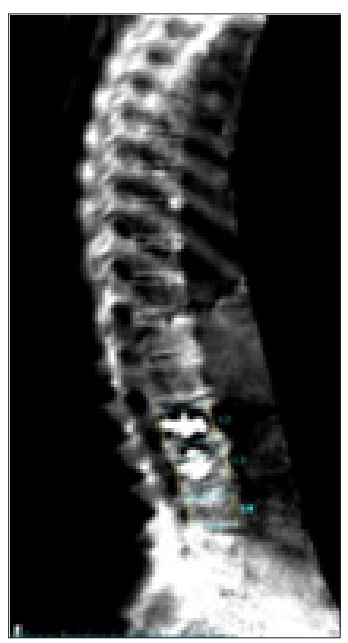

Figure 13:

\section{Conclusion}

DMAB- therapy (Prolia ${ }^{\circledR)}$ is a safe and highly effective treatment option for osteoporosis. It is characterized by a sustained 
reduction in the risk of fractures in a group of high-risk patients with very good tolerability. It is characterized by a reliable and sustained reduction of the bone remodeling rate and a verifiable continuous increase in bone mineral content (BMD). The annual incidence of new vertebral and non-vertebral fractures remains low with constant DMAB- therapy. However, the indication for the use of DAMB must be carefully considered and communicated to patients due to the potential complications (ONJ, hypocalcemia, increased fracture incidence after discontinuation of therapy). If therapy is discontinued or paused, there is a risk of loss of BMD, which in individual cases can still fall below the initial level before therapy begins!

\section{References}

1. Defer A (2016) Stellenwert der erfassung von kraft und balance für das sturz- und frakturrisiko älterer frauen. Osteologie 1: 27-30.

2. Hadji P (2014) Baseline-daten einer prospektiven beobachtungsstudie zum einnahme-verhalten von medikamenten bei frauen mit PMO, die Denosumab erhalten. Osteologie Suppl 1: 34-35.
3. Hadji P (2013) The epidemiology of osteoporosis - Bone Evaluation Study (BEST): an analysis of routine health insurance data. Dtsch Arztebl Int 110(4): 52-57.

4. Papapoulus S (2015) The effect of 8 or 5 years of denosumab treatment in postmenopausal woman with osteoporosis: results from the FREEDOM Extension study. Osteoporos Int 26(12): 2773-2783.

5. Mikosch P (2011) Osteoporosetherapie mit denosumab: 6-Jahresdaten zu knochendichte, knochenumsatz und verträglichkeit. J Miner Stoffwechs 18(1): 56-57.

6. Bone HG (2015) Ten years of denosumab treatment in postmenopausal woman with osteoporosis: Results from the FREEDOM extension trial. J Bone Miner Res 30(1): 471.

7. Tuncel $\mathrm{T}$ (2017) Compliance von osteoporosepatienten ohne zusätzliche schulungsmaßnahmen. Osteologie 2: 100-104.

8. Anastasilakis AD (2017) Clinical features of 24 patients with reboundassociated vertebral fractures after denosumab discontinuation: Systematic review and additional cases. J Bone Miner Res 32(6): 12911296.

9. Aubry-Rozier B (2016) Severe spontaneous vertebral fractures after denosumab discontinuation: three case reports. Osteoporos Int 27(5): 1923-1925. 